\title{
Time-Dependent and/or Nonlocal Representations of Hilbert Spaces in Quantum Theory
}

\author{
M. Znojil
}

\begin{abstract}
A few recent innovations of the applicability of standard textbook Quantum Theory are reviewed. The three-Hilbert-space formulation of the theory (known from the interacting boson models in nuclear physics) is discussed in its slightly broadened four-Hilbert-space update. Among applications involving several new scattering and bound-state problems the central role is played by models using apparently non-Hermitian (often called "crypto-Hermitian") Hamiltonians with real spectra. The formalism (originally inspired by the topical need for a mathematically consistent description of tobogganic quantum models) is shown to admit even certain unusual nonlocal and/or "moving-frame" representations $\mathcal{H}^{(S)}$ of the standard physical Hilbert space of wave functions.
\end{abstract}

Keywords: Quantum Theory, cryptohermitian operators of observables, stable bound states, unitary scattering, quantum toboggans, supersymmetry, time-dependent models.

\section{Introduction}

The Fourier transformation $\mathcal{F}: \psi(x) \rightarrow \tilde{\psi}(p)$ of wave functions converts differential kinetic-energy operator $K \sim \mathrm{d}^{2} / \mathrm{d} x^{2}$ into a trivial multiplication by a number, $\tilde{K}=\mathcal{F} K \mathcal{F}^{-1} \sim p^{2}$. This means that for certain quantum systems the Fourier transformation offers a simplification of the solution of the Schrödinger equation. The generalized, nonunitary (often called Dyson) mappings $\Omega$ play the same simplifying role in the context of nuclear physics [1]. In our present brief review paper we intend to recall and discuss very recent progress and, mainly, a few of our own results in this direction.

Our text will be more or less self-contained, though the limitations imposed on its length will force us to skip all remarks on the history of the subject as well as on references and on a broader context. Fortunately, interested readers may very easily get acquainted with these aspects of the new theory in several very thorough and extensive reviews [2] and also in our own recent compact review [3] and/or in our two-years-old short paper [4].

In section 2 we shall start our discussion from bound-state models characterized by the loss of observability of complexified coordinates. In the generic dynamical scenario where the Riemann surface of the wave functions can be assumed multisheeted, we shall define certain monodromy-sensitive models called quantum toboggans. Our selection of their sample applications will cover innovative models possessing several branch points in the complex $x$-plane and/or exhibiting supersymmetry.

Section 3 will offer information about the specific cryptohermitian approach to bound-state models characterized by the manifest time-dependence of their op- erators of observables (cf. paragraph 3.1) or by the presence of a fundamental length in the theory (cf. paragraph 3.2).

The two possible mechanisms of a return to unitarity in the models of scattering by complex potentials will be described in Section 4 . Via concrete examples we shall emphasize the beneficial role of a "smearing" of phenomenological potentials and the necessity of an appropriate redefinition of the effective mass in certain regimes.

Section 5 contains a few concluding remarks. For the sake of completeness, a few technical remarks concerning the role of the Dyson mapping in the abstract formulation of Quantum Theory as well as in some of its concrete applications will be added in the form of three Appendices.

\section{Quantum theories working with quadruplets of alternative Hilbert spaces}

Within the cryptohermitian approach, a new category of models of bound states appeared, a few years ago, under the name of quantum toboggans [5]. Their introduction extended the class of integration paths of complexified "coordinates" $x=q(s)$ in the standard Schrödinger equations to certain topologically nontrivial complex trajectories. The Hamiltonians $H^{(T)}=p^{2}+V^{(T)}(x)$ containing analytic potentials $V^{(T)}(x)$ with singularities (the superscripts $(T)$ stand here for "tobogganic") were connected with the generalized complex asymptotic boundary conditions and specified as operating in a suitable Hilbert space $\mathcal{H}^{(T)}$ of wave functions 
in which the Hamiltonian itself is manifestly nonHermitian.

Practical phenomenological use of any cryptohermitian quantum model requires, firstly, a sufficiently persuasive demonstration of the reality of its spectrum and, secondly, the availability of at least one metric operator $\Theta=\Theta(H)$ (cf. Appendices A-C for its definition). Usually, both of these conditions are nontrivial, so that any form of the solvability of the model is particularly helpful. Vice versa, once the Hamiltonian $H$ proves solvable in Hilbert space $\mathcal{H}^{(T)}$, we may rely upon the availability of the closed solutions of the underlying Schrödinger equations and on the related specific spectral representations of the necessary operators (cf. [6, 7] for more details).

The topological nontrivality of the tobogganic paths of coordinates running over several Riemann sheets of wave functions happened to lead to severe complications in the numerical attempts to compute the spectra. This difficulty becomes almost insurmountable when the wave functions describing quantum toboggans happen to possess two or more branch points (cf. [8] for an illustrative example). For these reasons it is recommended to rectify the tobogganic integration paths via a suitable change of variables in a preparatory step [9]. Our tobogganic Schroedinger equations then acquire the generalized eigenvalueproblem form $H \psi=E W \psi$ of the so called SturmSchroedinger equations with the rectified Hamiltonian $H \neq H^{\dagger}$ and with a nontrivial weight operator $W \neq W^{\dagger} \neq I$. Both of these operators are defined in another, transformed, "more friendly" Hilbert space $\mathcal{H}^{(F)}$ of course [10].

\subsection{Supersymmetric quantum toboggans}

The introduction of the cryptohermitian and tobogganic models proved useful in the context of supersymmetry (SUSY). A sample of papers devoted to this subject is referenced in [2]. The easiest case (called supersymmetric quantum mechanics) uses just the Hamiltonian and the two charge operators generating the SUSY algebra,

$$
\begin{aligned}
& \mathcal{H}=\left[\begin{array}{cc}
H^{(-)} & 0 \\
0 & H^{(+)}
\end{array}\right]=\left[\begin{array}{cc}
B A & 0 \\
0 & A B
\end{array}\right], \\
& \mathcal{Q}=\left[\begin{array}{ll}
0 & 0 \\
A & 0
\end{array}\right], \quad \tilde{\mathcal{Q}}=\left[\begin{array}{cc}
0 & B \\
0 & 0
\end{array}\right] .
\end{aligned}
$$

For the solvable model of ref. [11] the energy spectrum (composed of four families $\left.E_{n}=a(n), \ldots, d(n)\right)$ is displayed in figure 1. At $\gamma=-1 / 2$ the singularity vanishes and the (up to the ground state) doubly degenerate SUSY spectrum becomes strictly equidistant.
The imposition of supersymmetry has been extended to quantum toboggans in [12]. Both the components of the super-Hamiltonian were defined along topologically nontrivial complex curves which connect several Riemann sheets of the wave function. The new feature of this generalized model lies in the non-uniqueness of the map $\mathcal{T}$ between "tobogganic" partner curves. As a consequence, we must redefine the creation- and annihilation-like operators as follows,

$$
\begin{aligned}
& A=-\mathcal{T} \frac{\mathrm{d}}{\mathrm{d} x}+\mathcal{T} W^{(-)}(x), \\
& B=\frac{\mathrm{d}}{\mathrm{d} x} \mathcal{T}^{-1}+W^{(-)}(x) \mathcal{T}^{-1}
\end{aligned}
$$

In contrast to the non-tobogganic cases, the Hermitian-conjugation operator $\mathcal{T}$ even ceases to be involutory (i.e., $\mathcal{T} \neq \mathcal{T}^{-1}$, cf. paper [12] for more details).

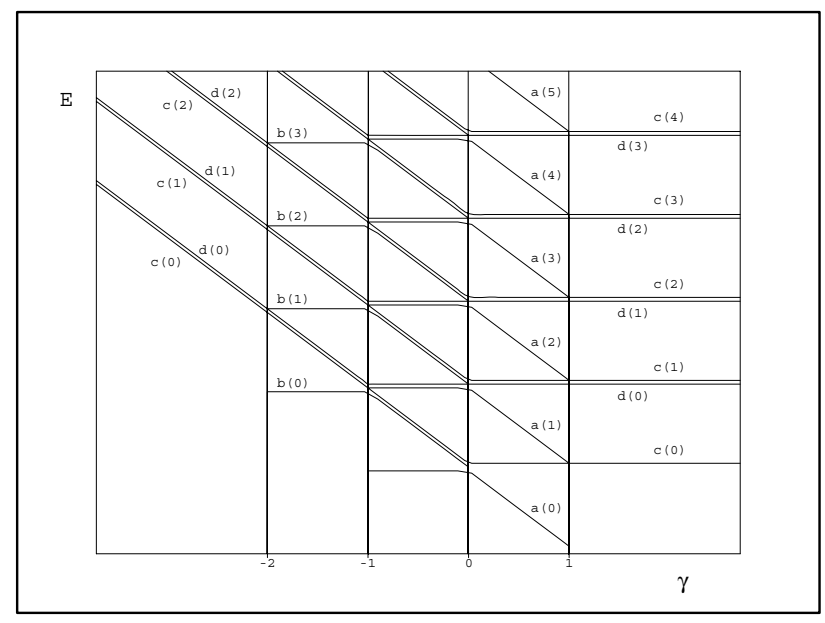

Fig. 1: Spectrum of the singular supersymmetric harmonic oscillator

\subsection{Four-Hilbert-space Quantum Mechanics}

In a way explained in our papers [7], the tobogganic quantum systems with real energies generated by their apparently non-Hermitian Hamiltonians may be assigned the entirely standard and consistent probabilistic interpretation. For this purpose the initial Hilbert space $\mathcal{H}^{(T)}$ is replaced by another, friendly Hilbert space $\mathcal{H}^{(F)}$ in which the above-mentioned Sturm-Schroedinger equations $H \psi=E W \psi$ have to be solved. This forces us to generalize the three-Hilbert-space scheme of paper [3] [cf. also Appendices A and B and figure 2] and to use the following four-Hilbert-space pattern of mappings 


\begin{tabular}{|c|}
\hline tobogganic space $\mathcal{H}^{(\mathrm{T})}$ \\
analytic multivalued $\psi[q(s)]$ \\
multisheeted paths $q^{(N)}(s)$
\end{tabular}

(the change of variables)

$\downarrow$ rectification

physics in $\mathcal{H}^{(\mathrm{P})}$
$\mathfrak{h}=\mathfrak{h}^{\dagger}, \quad \mathfrak{w}=\mathfrak{w}^{\dagger}$
dynamics via topology
$\uparrow \quad \begin{gathered}\text { (the unitary mapping) } \\ \text { equivalence }\end{gathered}$

feasibility in $\mathcal{H}^{(\mathrm{F})}$
$H \neq H^{\dagger}, \quad W \neq W^{\dagger}$
Sturm - Schrödinger eqs.

\section{(metric is introduced) hermitization}

standard space $\mathcal{H}^{(\mathrm{S})}$
$H=H^{\ddagger}, \quad W=W^{\ddagger}$
ad hoc metric $\Theta \neq I$
The analyticity of the original wave function $\psi[q(s)]$ along the given tobogganic integration path with parameter $s \in(-\infty, \infty)$ is assumed. The rectification transition between Hilbert spaces $\mathcal{H}^{(T)}$ and $\mathcal{H}^{(F)}$ is tractable as an equivalence transformation under this assumption [10]. In the subsequent sequence of maps $F \rightarrow S$ and $F \rightarrow P$ one simply follows the old three-Hilbert-space pattern of Appendix C [3] in which just the nontrivial weight operators $W$ and/or w are added and appear in the respective generalized SturmSchrödinger equations.

Marginally, let us add that various, suitably modified spectral representations of the eligible metric operators may be used, say, in the form derived in [7]. The purely kinematical and exactly solvable topologically nontrivial "quantum knot" example of ref. [13] can also be recalled here as an exactly solvable illustration in which the confining role of the traditional potential is fully simulated by the mere topologically nontrivial shape of the complex integration path.

\section{Bound-state theories working with the triplets of alternative Hilbert spaces}

\subsection{Quantum models admitting the time-dependence of their cryptohermitian Hamiltonians}

In our review [3] of the three-Hilbert-space (3HS) formalism we issued a warning that some of the consequences of the enhanced flexibility of the language and definitions may sound like new paradoxes. For illustration, let us mention just that in the 3HS approach the generator $H_{\text {(gen) }}=H_{\text {(gen) }}(t)$ of the time-evolution of wave functions is allowed to be different from the Hamiltonian operator $H=H(t)$ of the system in question [14].

The key to the disentanglement of the similar puzzles is easily found in the explicit specification of the Hilbert space in which we define the Hermitian conjugation. We showed in [14] that the use of the full triplet of spaces of figure 2 becomes unavoidable whenever our cryptohermitian observables are assumed time-dependent because their variations may and must be matched by the time-dependence of the representation of the physical ad hoc Hilbert space $\mathcal{H}^{(S)}$. Its nontrivial inner product is capable of playing the role of a "moving frame" image of the original physical Hilbert space $\mathcal{H}^{(P)}$. Although our "true" Hamiltonian (i.e., operator $\mathfrak{h}(t)$ in $\mathcal{H}^{(P)}$ ) is the generator of the time evolution in $\mathcal{H}^{(P)}$, the time-evolution of the wave functions in $\mathcal{H}^{(S)}$ is controlled not only by the "dynamical" influence of $H=H(t)$ itself but also by the "kinematical" influence of the time-dependence of the "rotating" Dyson mapping $\Omega=\Omega(t)$. Thus, the existence of any other given and manifestly timedependent observable $\mathfrak{o}(t)$ in $\mathcal{H}^{(P)}$ will leave its trace in Dyson map $\Omega(t)$, i.e., in metric $\Theta(t)$, i.e., in the time-dependence of the "moving frame" Hilbert space $\mathcal{H}^{(S)}$.

This circumstance implies the existence of two pullbacks of the evolution law from $\mathcal{H}^{(P)}$ to $\mathcal{H}^{(S)}$, with the recipe $|\varphi(t)\rangle=\Omega^{-1}(t) \mid \varphi(t) \succ$ being clearly different from the complementary recipe $\langle\langle\varphi(t)|=\prec \varphi(t)| \Omega(t)$. The same Dyson mapping leads to the two different evolution operators, viz., to the evolution law for kets,

$$
|\varphi(t)\rangle=U_{R}(t)|\varphi(0)\rangle, \quad U_{R}(t)=\Omega^{-1}(t) u(t) \Omega(0)
$$

and to the different evolution law for brabras,

$$
\left.|\varphi(t)\rangle\rangle=U_{L}^{\dagger}(t)|\varphi(0)\rangle\right\rangle, \quad U_{L}^{\dagger}(t)=\Omega^{\dagger}(t) u(t)\left[\Omega^{-1}(0)\right]^{\dagger} .
$$

We have no space here for the detailed reproduction of the whole flow of this argument as presented in [14]. Its final outcome is the definition of the common timeevolution generator

$$
H_{(g e n)}(t)=H(t)-\mathrm{i} \Omega^{-1}(t) \dot{\Omega}(t) .
$$

entering the final doublet of time-dependent Schrödinger equations

$$
\begin{aligned}
\mathrm{i} \partial_{t}|\Phi(t)\rangle & =H_{(g e n)}(t)|\Phi(t)\rangle, \\
\left.\mathrm{i} \partial_{t}|\Phi(t)\rangle\right\rangle & \left.=H_{(g e n)}(t)|\Phi(t)\rangle\right\rangle .
\end{aligned}
$$

This ultimately clarifies the artificial character and redundancy of the Mostafazadeh's conjecture [15] of 
quasistationarity, i.e., of the requirement of timeindependence of the inner products and of the metric, i.e., ipso facto, of Hilbert space $\mathcal{H}^{(S)}$.

\subsection{Systems admitting a controllable nonlocality}

In a way emphasized by Jones [16] the direct observability of coordinates $x$ is lost for the majority of the parity-times-time-reversal-symmetric (or, briefly, $\mathcal{P} \mathcal{T}$ symmetric) quantum Hamiltonians. In the context of scattering, this forced us to admit a non-locality of the potentials in [17]. Fortunately, in the context of bound states the loss of the observability of coordinates is much less restrictive since we do not need to prepare any asymptotically free states. The admissible Hilbert-space metrics $\Theta$ may be chosen as moderately non-local acquiring, in the simplest theoretical scenario as proposed in our paper [18], the form of a short-ranged kernel in a double-integral normalization or in the inner products of the wave functions. The standard Dirac's delta-function kernel is simply reobtained in the zero-range limit.

In refs. $[17,19]$ we proposed several bound-state toy models exhibiting, in a confined-motion dynamical regime, various forms of an explicit control of the measure $\theta$ of their dynamically generated non-locality. The exact solvability of some of these models even allowed us to assign each Hamiltonian the complete menu of its hermitizing metrics $\Theta=\Theta_{\theta}$ distinguished by their optional fundamental lengths $\theta \in(0, \infty)$. In this setting the local metrics reappear at $\theta=0$ while certain standard hermitizations only appeared there as infinitely long-ranged, with $\theta=\infty$.

\section{Scattering theories using pairs of Hilbert spaces $\mathcal{H}^{(P)} \neq \mathcal{H}^{(F)}$}

In our last illustrative application of $3 \mathrm{HS}$ formalism, let us select just two non-equivalent Hilbert spaces $\mathcal{H}^{(F, S)}$ and turn to scattering theory where one assumes that the coordinate is certainly measurable/measured at large distances. This means that we may employ the operators in coordinate representation and accept only such models where the metric operator remains asymptotically proportional to delta function, $\left\langle x|\Theta| x^{\prime}\right\rangle \sim \delta\left(x-x^{\prime}\right)$ at $|x| \gg 1$ and $\left|x^{\prime}\right| \gg 1$. A few concrete models of this type were described in refs. [17, 19] using minimally nonlocal, "smeared" point interactions of various types (which were, in the latter case, multi-centered). The use of nonperturbative discretization techniques rendered possible the construction of the (incidentally, unique) metric $\Theta$ compatible with the required asymptotic locality.

The resulting physical picture of scattering was unitary and fully compatible with our intuitive expec- tations. In our last paper [20] the scope of the theory has further been extended to the generalized scattering models, where the matrix elements $\left\langle x|\Theta| x^{\prime}\right\rangle$ of the metric were allowed operator-valued.

A slightly different approach to scattering has been initiated in paper [21] where we studied the analytic and "realistic" Coulombic cryptohermitian potentials defined along U-shaped complex trajectories circumventing the origin in the complex $x$ plane from below. Unfortunately, this model was unstable with respect to perturbations. A few years later we clarified, in paper [22], that a very convenient stabilization of the model may be based on a minus-sign choice of the bare mass in the Schrödiner equation. Very soon afterwards we also revealed that the scattering by the amended Hamiltonian is unitary [23]. The transmission and reflection coefficients were evaluated in closed analytic form exhibiting the coincidence of the bound-state energies with the poles of the transmission coefficients. Thus, after a moderate modification a number of observations forming the analytic theory of S-matrix has been found transferrable to the cryptohermitian quantum theory.

\section{Conclusions}

One of paradoxes characterizing Quantum Theory may be seen in the contrast between its stable status in experiments (where, typically, its first principles are appreciated as unexpectedly robust [24]) and its fragile status in the mathematical context, where virtually all of its rigorous formulations are steadily being found, for this or that reason, not entirely satisfactory [25]. In fact, at least a part of this apparent conflict is just a pseudoconflict. Its roots can be traced back to various purely conceptual misunderstandings. In our present review we emphasized that within the comparatively narrow framework of quantum theory using cryptohermitian representations of observables the majority of these misunderstandings can be clarified, mostly via a careful use of an adequate notation.

The core of our present message can be seen in the unified outline of the resolution of the internetmediated debate (cf. [3] for references) in which the admissibility and consistent tractability of the manifestly time-dependent cryptohermitian observables has been questioned. It is now clear that the reduction of the scope of the theory to the mere quasistationary systems as proposed by Mostafazadeh [15] is unfounded.

This bound-state-related message can be seen accompanied by the clarification of a return to unitarity in the models of scattering mediated by cryptohermitian interactions. The currently valid conclusion is that it makes sense to combine the complexification of the short-range interactions with our making them at least slightly nonlocal. We have seen that, in parallel, also the metric can be required to exhibit a certain limited degree of nonlocality. 
New questions emerge in this context. This means that in spite of all the recent rapid progress the current intensive development of the cryptohermitian quantum theory is still fairly far from its completion.

\section{Appendix A: Hilbert space in our present notation}

In our review paper [3] we explained that one of the most natural formulations of the abstract Quantum Theory should follow the ideas of Scholtz et al [1] by constructing the three parallel representatives of any given wave function living in the three separate Hilbert spaces. We argued that the use of the three-Hilbertspace (3HS) formulation of Quantum Theory seems best capable of clarifying a few paradoxes emerging in connection with the concept of Hermiticity and encountered in the recent literature. We emphasized in [3] that many quantum Hamiltonians with real spectra, characterized by their authors as manifestly nonHermitian, should and must be re-classified as Hermitian. In this sense we fully accepted the dictum of standard textbooks on quantum theory and complemented the corresponding postulates just by a few explanatory comments.

In a brief summary of this argument let us recall that the states $\psi$ of a (say, one-dimensional) quantum system are often assumed represented by normalized elements of the simplest physical and computation friendly concrete Hilbert space $\mathbb{L}^{2}(\mathbb{R})$. This is already just a specific assumption with restrictive consequences. Thus, in a more ambitious picture of a general quantum system each state $\psi$ should only be perceived as an element $|\psi\rangle$ of an abstract vector space $\mathcal{V}$. The equally abstract dual vector space $\mathcal{V}^{\prime}$ of linear functionals over $\mathcal{V}$ may be bigger, $\mathcal{V}^{\prime} \supset \mathcal{V}$. In the most common selfdual case with $\mathcal{V}^{\prime}=\mathcal{V}$ one speaks about the Hilbert space $\mathcal{H}^{(F)}:=\left(\mathcal{V}, \mathcal{V}^{\prime}\right)$ where the superscript ${ }^{(F)}$ stands, say, for (user-)friendly or "feasible".

In many standard formulations of the first principles of Quantum Theory the well known Dirac's braket notation is used, with $|\psi\rangle \in \mathcal{V}$ and $\langle\psi| \in \mathcal{V}^{\prime}$ for a fixed or "favored" Hilbert space $\mathcal{H}^{(F)}$. At the same time, this choice of the notation does not exclude a transition (say, $\Omega$ ) to some other vector and Hilbert spaces denoting, e.g., $\Omega|\psi\rangle:=\mid \psi \succ \in \mathcal{W}$ and using here the slightly deformed, spiked ket symbols [3].

\section{Appendix B: Dyson mapping $\Omega$ as a nonunitary generalization of the Fourier transformation $\mathcal{F}$}

In the context of nuclear physics the use of the single, favored Hilbert space $\mathcal{H}^{(F)}$ is rather restrictive. For example, in the context of the so called inter- acting boson model and in the way inspired by the well known advantages of the use of the usual unitary Fourier transformation $\mathcal{F}=\left[\mathcal{F}^{\dagger}\right]^{-1}$, nuclear physicists discovered that their constructive purposes may be much better served by a suitable generalized, manifestly non-unitary (often called Dyson) invertible mapping $\Omega$.

More details may be found in paper [1], where the operators $\Omega$ were described as mediating the transition from a friendly bosonic vector space $\mathcal{V}$ into another, fermionic and "physical" vector space $\mathcal{W}$. The deepened mathematical differences between "bosonic" (i.e., simpler) $\mathcal{V}$ and fermionic (i.e., complicated, computationally much less accessible) $\mathcal{W}$ weakens the parallelism between $\Omega$ and $\mathcal{F}$ since the latter operator merely switches between the so called coordinate- and momentum-representations of $\psi$ s lying in the same Hilbert space $\mathbb{L}^{2}(\mathbb{R})$.

This encouraged us to propose, in [3], visual identification of the bras and kets in one-to-one correspondence to the space in which they live, with $|\psi\rangle \in \mathcal{V}$ while $\Omega|\psi\rangle:=\mid \psi \succ \in \mathcal{W}$. For duals (i.e., bra-vectors) we recommended the same notation, with $\langle\psi| \in \mathcal{V}^{\prime}$ while $\left\langle\psi\left|\Omega^{\dagger}:=\prec \psi\right| \in \mathcal{W}^{\prime}\right.$.

\section{Appendix C: The connection between Dyson map $\Omega$ and metric $\Theta$}

In the notation of Appendix B one represents the same state $\psi$ in two non-equivalent Hilbert spaces, viz., in the friendly F-space $\mathcal{H}^{(F)}:=\left(\mathcal{V}, \mathcal{V}^{\prime}\right)$ and in the physical P-space $\mathcal{H}^{(P)}:=\left(\mathcal{W}, \mathcal{W}^{\prime}\right)$ (characterized by the "spiked" kets and bras). The latter space is, by construction, manifestly non-equivalent to the former one since, by definition, we have, for overlaps, $\prec \psi_{a} \mid \psi_{b} \succ=\left\langle\psi_{a}\left|\Omega^{\dagger} \Omega\right| \psi_{b}\right\rangle \neq\left\langle\psi_{a} \mid \psi_{b}\right\rangle$.

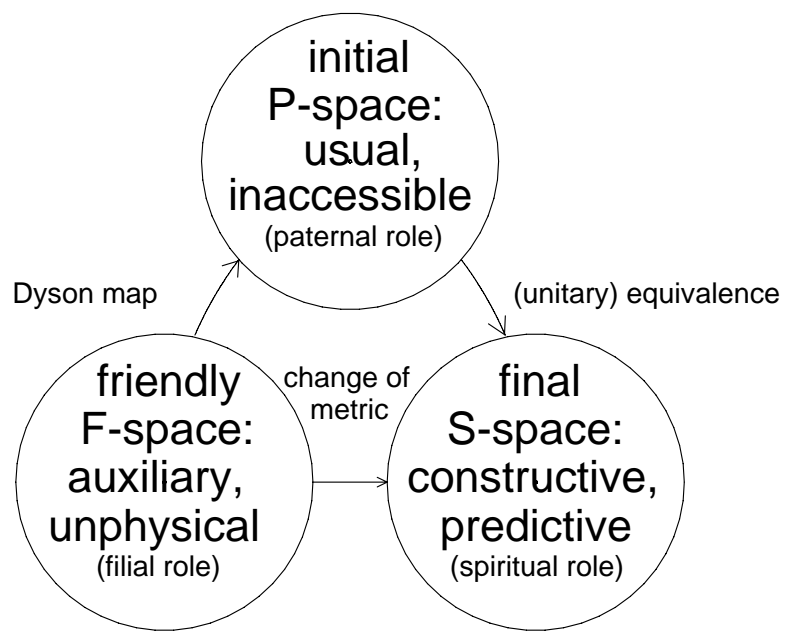

Fig. 2: The same physics is predicted in $\mathcal{H}^{(P)}$ and in $\mathcal{H}^{(S)}$ while, presumably, the calculations are all performed in $\mathcal{H}^{(F)}$ 
According to our review [3], the demonstration of unitary non-equivalence between $\mathcal{H}^{(F)}$ and $\mathcal{H}^{(P)}$ can easily be converted into a proof of unitary equivalence between $\mathcal{H}^{(P)}$ and another, third, standardized Hilbert space $\mathcal{H}^{(S)}:=\left(\mathcal{V}, \mathcal{V}^{\prime \prime}\right)$. Indeed, we are free to introduce a redefined vector space of linear functionals $\mathcal{V}^{\prime \prime}$ such that the equivalence will be achieved. For the latter purpose it is sufficient to introduce the special duals $\left\langle\langle\psi| \in \mathcal{V}^{\prime \prime}\right.$ denoted by the new, "brabra" Diracinspired symbol. In terms of a given Dyson operator $\Omega$ we may define these brabras, for the sake of definiteness, by the formula $\left\langle\left\langle\psi_{a}\right|=\left\langle\psi_{a}\right| \Theta\right.$ of ref. [6], where we abbreviated $\Theta=\Omega^{\dagger} \Omega$.

In [1] the new operator $\Theta$ has been called metric. It defines the inner products in the "second auxiliary" (i.e., in its nuclear-physics exemplification, second bosonic) Hilbert space $\mathcal{H}^{(S)}$ which is, by construction, unitarily equivalent to the original physical $\mathcal{H}^{(P)}$. The whole $3 \mathrm{HS}$ scheme is given a compact graphical form in figure 2 .

\section{Acknowledgement}

This work has been supported by MŠMT Doppler Institute project LC06002, by Institutional Research Plan AV0Z10480505 and by GAČR grant 202/07/1307.

\section{References}

[1] Scholtz, F. G., Geyer, H. B., Hahne, F. J. W.: Quasi-Hermitian Operators in Quantum Mechanics and the Variational Principle, Ann. Phys., Vol. 213 (1992) p. 74-101.

[2] Dorey, P., Dunning, C., Tateo, R.: The ODE/IM Correspondence, J. Phys. A: Math. Theor., Vol. 40 (2007) p. R205-R283 (arXiv:hepth/0703066).

Bender, C. M.: Making Sense of non-Hermitian Hamiltonians, Rep. Prog. Phys., Vol. 70 (2007) p. 947-1018 (arXiv: hep-th/0703096);

Mostafazadeh, A.: Pseudo-Hermitian Quantum Mechanics, arXiv:0810.5643.

[3] Znojil, M.: Three-Hilbert-space formulation of Quantum Mechanics, SIGMA, Vol. 5 (2009), 001, 19 pages (arXiv:0901.0700).

[4] Znojil, M.: PT-symmetric quantum chain models, Acta Polytechnica, Vol. 47 (2007) p. 9-14.

[5] Znojil, M.: PT-symmetric quantum toboggans. Phys. Lett. A, Vol. 342 (2005) 36-47.

[6] Znojil, M.: On the Role of Normalization Factors and Pseudometric in Crypto-Hermitian Quantum Models, SIGMA 4 (2008), p. 001, 9 pages (arXiv: 0710.4432).
[7] Znojil, M.: Identification of observables in quantum toboggans. J. Phys. A: Math. Theor. 41 (2008) 215304 (arXiv:0803.0403);

Znojil, M., Geyer, H. B.: Sturm-Schroedinger equations: formula for metric. Pramana J. Phys., Vol. 73 (2009), p. 299-306 (arXiv:0904.2293).

[8] Znojil, M.: Quantum toboggans with two branch points. Phys. Lett. A, Vol. 372 (2008) p. 584-590 (arXiv: 0708.0087).

[9] Novotný, J.: http://demonstrations.wolfram.com/ TheQuantum TobogganicPaths

[10] Znojil, M.: Quantum toboggans: models exhibiting a multisheeted PT symmetry. J. Phys.: Conference Series, Vol. 128 (2008) 012046.

[11] Znojil, M.: Non-Hermitian SUSY and singular, PT-symmetrized oscillators. J. Phys. A: Math. Gen., Vol. 35 (2002) p. $2341-2352$ (arXiv:hepth/0201056).

[12] Znojil, M., Jakubský, V.: Supersymmetric quantum mechanics living on topologically nontrivial Riemann surfaces. Pramana J. Phys., Vol. 73 (2009) p. 397-404.

[13] Znojil, M.: Quantum knots. Phys. Lett. A, Vol. 372 (2008) p. 3591-3596.

[14] Znojil, M.: Time-dependent version of cryptohermitian quantum theory. Phys. Rev. D, Vol. 78 (2008) 085003 (arXiv:0809.2874).

[15] Mostafazadeh, A.: Time-dependent pseudoHermitian Hamiltonians defining a unitary quantum system and uniqueness of the metric operator. Phys. Lett. B, Vol. 650 (2007) p. 208-212.

[16] Jones, H. F.: Scattering from localized nonHermitian potentials. Phys. Rev. D, Vol. $\mathbf{7 6}$ (2007) 125003 .

[17] Znojil, M.: Scattering theory with localized non-Hermiticities. Phys. Rev. D, Vol. 78 (2008) 025026 (arXiv:0805.2800).

[18] Znojil, M.: Fundamental length in quantum theories with PT-symmetric Hamiltonians. Phys. Rev. D, Vol. 80 (2009) 045022 (13 pages, arXiv:0907.2677).

[19] Znojil, M.: Discrete PT-symmetric models of scattering. J. Phys. A: Math. Theor., Vol. 41 (2008) 292002 (arXiv:0806.2019);

Znojil, M.: Scattering theory using smeared non-Hermitian potentials. Phys. Rev. D, Vol. 80 (2009) 045009 (12 pages, arXiv:0903.1007).

[20] Znojil, M.: Cryptohermitian picture of scattering using quasilocal metric operators. SIGMA, Vol. 5 (2009) 085 (21 pages, arXiv:0908.4045). 
[21] Znojil, M., Lévai, G.: The Coulomb - harmonicoscillator correspondence in PT symmetric quantum mechanics. Phys. Lett. A, Vol. 271 (2000) p. 327-333.

[22] Znojil, M., Siegl, P., Lévai, G.: Asymptotically vanishing $\mathrm{PT}$-symmetric potentials and negativemass Schroedinger equations. Phys. Lett. A, Vol. 373 (2009) 1921-1924.

[23] Lévai, G., Siegl, P., Znojil, M.: Scattering in the PT-symmetric Coulomb potential. J. Phys. A: Math. Theor., Vol. 42 (2009) 295201 (9 pp, arXiv:0906.2092).

[24] Styer, D. F. et al.: Nine Formulations of Quantum Mechanics, Amer. J. Phys., Vol. 70 (2002), p. 288-297.
[25] Strocchi, F.: An Introduction to the Mathematical Structure of Quantum Mechanics. Singapore : World Scientfic, 2008 (2nd edition).

[26] Rosinger, E. E.: Deficient Mathematical Models of Quantum Theory. arXiv:quant-ph/0510138, and Mathematics and The Trouble with Physics, How Deep We Have to Go? arXiv:0707.1163.

Miloslav Znojil, DrSc.

Phone: +420266173286

E-mail: znojil@ujf.cas.cz

Nuclear Physics Institute

Academy of Science of the Czech Republic 25068 Řež, Czech Republic 